\title{
Comparison of dexmedetomidine with propofol as sedatives for pediatric patients undergoing magnetic resonance imaging: A meta-analysis of randomized controlled trials with trial sequential analysis
}

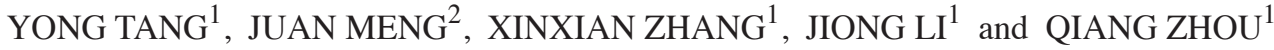 \\ ${ }^{1}$ Department of Radiology; ${ }^{2}$ Operating Room, Xuzhou Children's Hospital, \\ Xuzhou Medical University, Xuzhou, Jiangsu 221006, P.R. China
}

Received September 14, 2018; Accepted March 23, 2019

DOI: $10.3892 /$ etm.2019.7751

\begin{abstract}
Dexmedetomidine and propofol are commonly used sedative agents in pediatric patients undergoing magnetic resonance imaging (MRI). The present meta-analysis aimed to compare dexmedetomidine with propofol in pediatric patients undergoing MRI using trial sequential analysis (TSA). The PubMed, Cochrane Library and Web of Knowledge databases were systematically searched for entries up to August 2018 for potential randomized controlled trials comparing dexmedetomidine with propofol in pediatric patients undergoing MRI. Data were extracted by two independent authors and analyzed using Revman version 5.2 software. Six trials involving 415 pediatric patients were included in the final analysis. A shorter recovery time $(\mathrm{P}<0.01)$ and onset time of sedation were identified for propofol compared with dexmedetomidine $(\mathrm{P}<0.01)$; however, there were no significant differences in the duration of sedation $(\mathrm{P}=0.37)$. Furthermore, pediatric patients receiving propofol were discharged sooner than those receiving dexmedetomidine $(\mathrm{P}=0.02)$. The incidence of failed sedation did not significantly differ between the two groups $(\mathrm{P}=0.81)$. Propofol induced a lower incidence of $5-\mathrm{min}(\mathrm{P}=0.03)$ and $10-\mathrm{min}$ Pediatric Anesthesia Emergence Delirium $(\mathrm{P}<0.01)$, but a higher incidence of desaturation $(\mathrm{P}<0.01)$. The duration of MRI was similar between the two groups $(\mathrm{P}=0.15)$. TSA indicated that the monitoring boundary was crossed by the cumulative $\mathrm{z}$ curve, providing supportive evidence for the shorter recovery
\end{abstract}

Correspondence to: Dr Xinxian Zhang, Department of Radiology, Xuzhou Children's Hospital, Xuzhou Medical University, 18 Sudi North Road, Xuzhou, Jiangsu 221006, P.R. China

E-mail: zhangxinxian2017@126.com

Abbreviations: MRI, magnetic resonance imaging; TSA, trial sequential analysis; RCT, randomized controlled trial; PAED, Pediatric Anesthesia Emergence Delirium

Key words: dexmedetomidine, propofol, meta-analysis, trial sequential analysis, magnetic resonance imaging time in the propofol group. Propofol is recommended for pediatric sedation during MRI, owing to shorter recovery time and onset of sedation time, as well as a faster discharge from hospital, and a lower incidence of PAED score $>10$, compared with dexmedetomidine. However, considering the possibility of desaturation, propofol should be used with caution.

\section{Introduction}

Magnetic resonance imaging (MRI) is a commonly used diagnostic modality in the pediatric population owing to its high resolution and absence of radiation exposure $(1,2)$. High-quality imaging facilitates accurate diagnosis, optimal treatment and the monitoring of therapeutic responses in pediatric patients. However, undergoing MRI procedures may be stressful, particularly for pediatric subjects (3), who tend to experience anxiety and distress prior to and during scanning. The long duration of examination, the noise generated during the process and the narrow confines of MRI devices occasionally lead to failed scans in pediatric patients (4). To obtain high-quality images in pediatric patients, sedation is administered to prevent patient movement and mitigate emotional discomfort.

Dexmedetomidine is an $\alpha_{2}$-adrenergic receptor agonist that is widely used for procedures requiring sedation of pediatric patients due to its sedative and analgesic characteristics $(5,6)$. Propofol is also an effective and highly popular sedative agent in pediatric patients $(7,8)$. Several randomized controlled trials (RCTs) have compared the two drugs in pediatric patients undergoing MRI. Koroglu et al (9) reported a shorter recovery time in pediatric patients who received propofol, with a comparable time to onset of sedation and duration of sedation between the two drugs. A study by Wu et al (10) indicated a shorter recovery and onset of sedation for propofol, which is consistent with the results of a previous meta-analysis by our group (11), which included RCTs and non-RCTs. However, in this previous meta-analysis, one RCT (12) was missed in the pooling of data, and a novel RCT (13) was recently published, thus prompting an updated meta-analysis on this topic. Accordingly, a new meta-analysis with trial sequential analysis (TSA) performed with. 


\section{Materials and methods}

Search strategy. The PubMed, Cochrane Library and Web of Knowledge databases were searched for entries up to August 2018 for potential trials comparing dexmedetomidine and propofol in pediatric patients undergoing MRI without restriction by study type or publication language, according to the Preferred Reporting Items for Systematic Reviews and Meta-Analyses statement (14). The following terms were used in the literature search with various combinations: 'Dexmedetomidine' AND 'propofol' AND ('magnetic resonance imaging' OR 'MRI') AND ('pediatric' OR 'children' OR 'child' OR 'adolescence').

Inclusion criteria. Two authors (YT and JM) independently assessed the trial eligibility. Any disagreement between the two authors was resolved by the senior author (XZ). Eligible trials were required to fulfill the following criteria: i) RCT; ii) comparison of dexmedetomidine and propofol; iii) subjects aged $<18$ years; and iv) subjects undergoing MRI.

Data extraction. Baseline variables were extracted from the eligible studies, including the following: Publication year, first author, study period, region, number of patinets, patient age, body weight, sex, MRI machines and details on the intervention. Sedation parameters were collected, including recovery time, onset time of sedation, duration of sedation, discharge time and failed sedation. Timeline parameters are depicted in Fig. 1. The incidence of adverse events with sufficient data for analysis was also recorded. Desaturation was defined as oxygen saturation ( $<93 \%$. The Pediatric Anesthesia Emergence Delirium scale (PAED) (15) was used to monitor the behavior of the pediatric patients. Patients with a PAED score of $>10$ points had a high risk of emergent delirium. The number of patients with a PAED score of $>10$ points at 5 and $10 \mathrm{~min}$ after drug discontinuation was recorded as 5-min and 10-min PAED.

Risk of bias assessment. The major domains of bias in each study were assessed according to the recommendations from the Cochrane risk of bias tool (16). In general, the studies were categorized as having low, high and unknown risk of bias regarding the following items: Random sequence generation, allocation concealment, blinding of participants and staff, blinding of outcome assessment, incomplete outcome data, selective outcome reporting and other bias.

Trial sequential analysis. Meta-analysis may lead to type I errors due to an increased risk of random errors when insufficient data are included and due to multiple testing with new trials. Trial sequential analysis (TSA) is a program that calculates the required information size for a meta-analysis with an adjusted threshold for statistical significance in the cumulative meta-analysis, which may control the risk of type I and II errors. When the cumulative $\mathrm{z}$ curve crosses the TSA monitoring boundary, a sufficient level of evidence is achieved for the anticipated intervention effect. If the $\mathrm{z}$ curve dose not cross any boundary and the required information size has not been reached, evidence for a definite conclusion is not sufficient. TSA software version 0.9 beta (Copenhagen Trial Unit,
Copenhagen, Denmark) was applied in the study to estimate the optimal sample size.

Statistical analysis. All the meta-analyses were performed using Revman 5.3 software (Nordic Cochrane Centre, Cochrane Collaboration), with relative risks (RR) or standard mean differences (SMD) with 95\% confidence intervals (CIs) calculated for dichotomous and continuous variables, respectively. Heterogeneity among the studies was explored by a standard Cochrane's Q test and $\mathrm{I}^{2}$. A fixed- or random-effects model was used to estimate the differences between groups in the case of absence or presence of heterogeneity among the studies included. Heterogeneity among the studies was assessed using $\mathrm{I}^{2}$ statistics with $\mathrm{I}^{2}>50 \%$ being considered to indicate significant heterogeneity. The fixed-effects model was selected when $\mathrm{I}^{2} \leq 50 \%$ and the random-effects model was used when $\mathrm{I}^{2}>50 \%$. $\mathrm{P}<0.05$ was considered to indicate statistical significance.

\section{Results}

Study search and characteristics. A flow diagram depicting the selection process of studies is provided in Fig. 2. The initial search yielded 141 records from the PubMed, Web of Knowledge and Cochrane Library databases, from which 97 duplicates were excluded. After review of titles and abstracts, 80 articles were excluded. A further 11 trials were removed, and the details are provided in Fig. 2 Finally, 6 studies $(9,10,12,13,17,18)$, from 5 countries, including Turkey, the USA, Singapore, India and China, involving 415 pediatric patients (207 receiving dexmedetomidine and 208 propofol) were included in the final analysis. The study characteristics are displayed in Table I. Only 2 of the studies indicated the study period. A total of 3 studies $(10,12,17)$ reported details of sedation induction. Sevoflurane and nitrous oxide in oxygen were used for inhalation induction. The administration methods of dexmedetomidine and propofol are also provided in Table I. The type of MRI device was described in two studies $(12,13)$. Details of sedation effects and adverse events are presented in Table II. This included data for recovery time, onset of sedation time, duration of sedation, discharge time, failed sedation, desatuation, 5 min PAED and 10 min PAED.

Risk of bias assessment. The risk of bias assessment of the included studies is provided in Table III. All of the studies, except 3 studies, maintained a good control of each domain. Wu et al (10), Watt et al (12) and Xiao et al (13) did not include any details of the random sequence generation. Furthermore, the study by Xiao et al (13) had an unclear risk of bias regarding allocation concealment.

Sedation outcomes. The results of the meta-analysis on sedation efficacy are provided in Table IV, which includes recovery time, onset of sedation time, duration of sedation, discharge time and incidence of failed sedation. The data from 6 trials indicated a shorter recovery time [SMD (95\% CI): 1.11 (0.55, 1.66), $\mathrm{P}<0.01, \mathrm{I}^{2}=85 \%$; Fig. 3] and onset of sedation time for propofol compared with dexmedetomidine [SMD (95\%CI): 1.44 (0.77, 2.12), $\mathrm{P}<0.01, \mathrm{I}^{2}=86 \%$; Fig. 4]. Pediatric patients who received propofol were discharged from hospital 


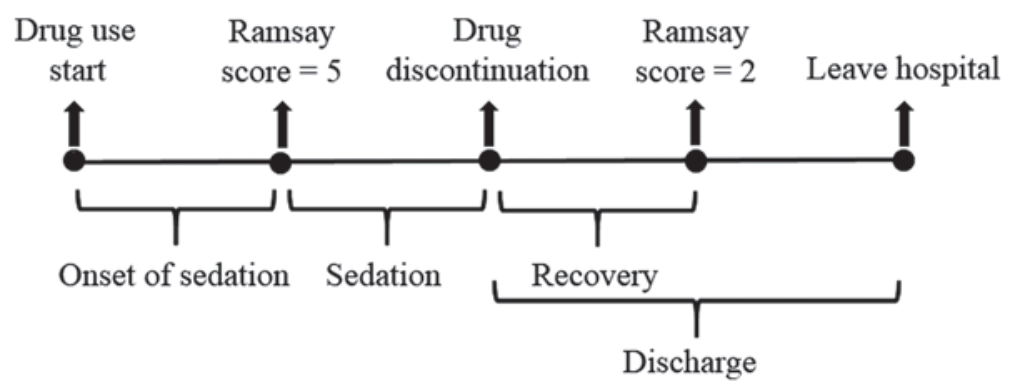

Figure 1. Depiction of timeline variables in the study.

\section{PRISMA 2009 flow diagram}
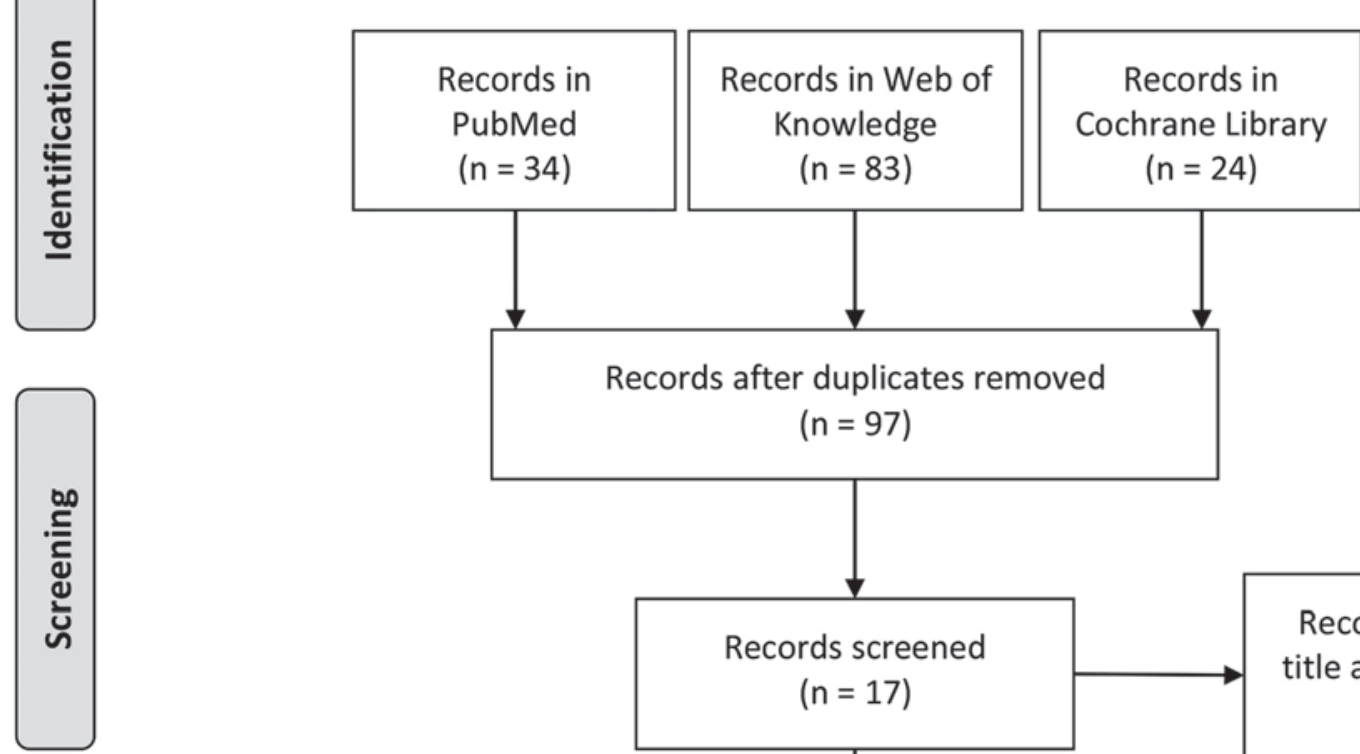

Records after duplicates removed

$$
(n=97)
$$
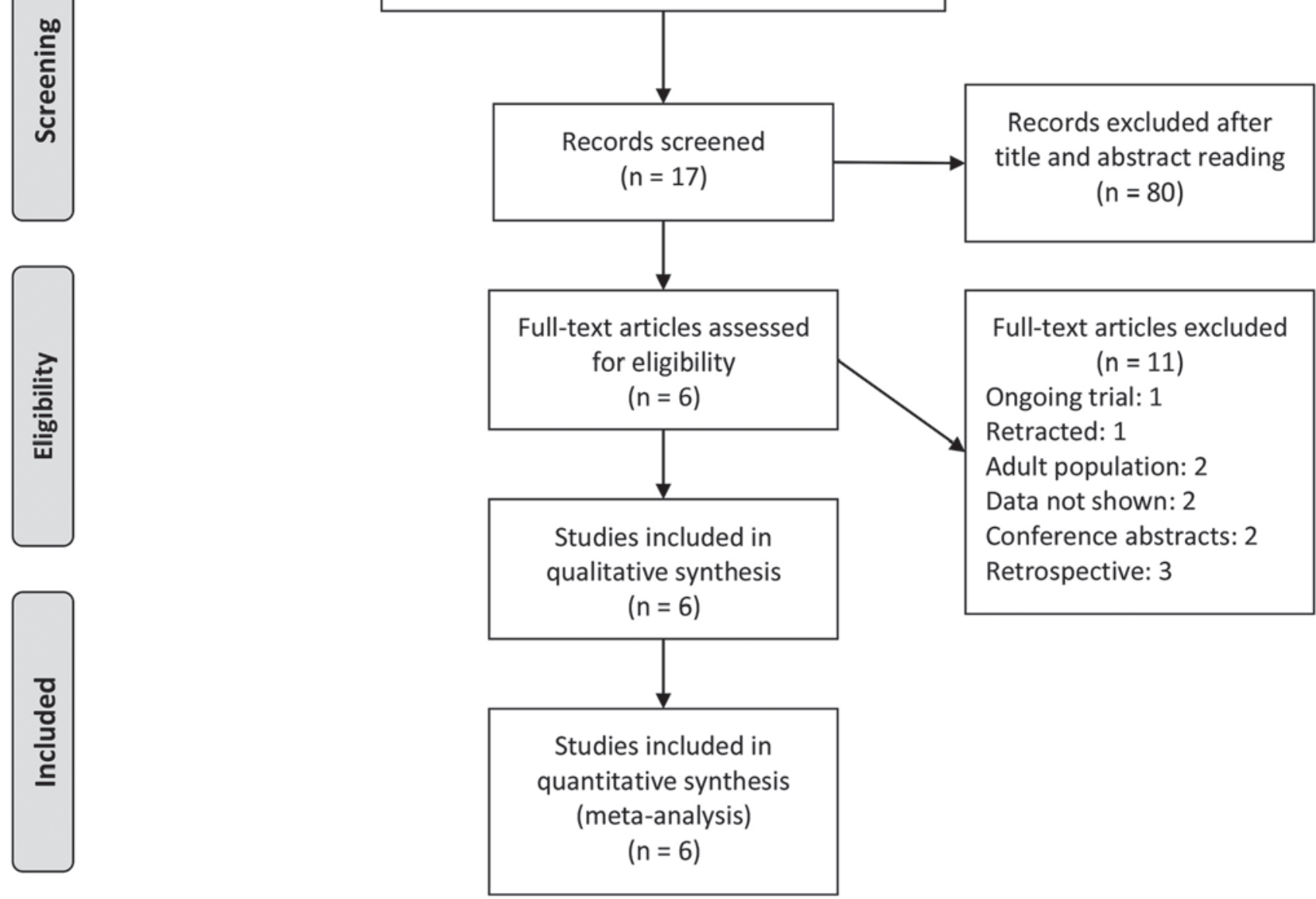

Full-text articles excluded $(n=11)$

Ongoing trial: 1

Retracted: 1

Adult population: 2

Data not shown: 2

Conference abstracts: 2

Retrospective: 3

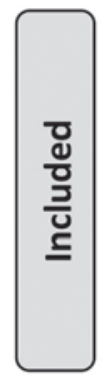

From: Moher D, Liberati A, Tetzlaff J, Altman DG, The PRISMA Group (2009). Preferred Reporting Items for Systematic Reviews and MetaAnalyses: The PRISMA Statement. PLoS Med 6(7): e1000097. doi:10.1371/journal.pmed1000097

For more information, visit www.prisma-statement.org. 


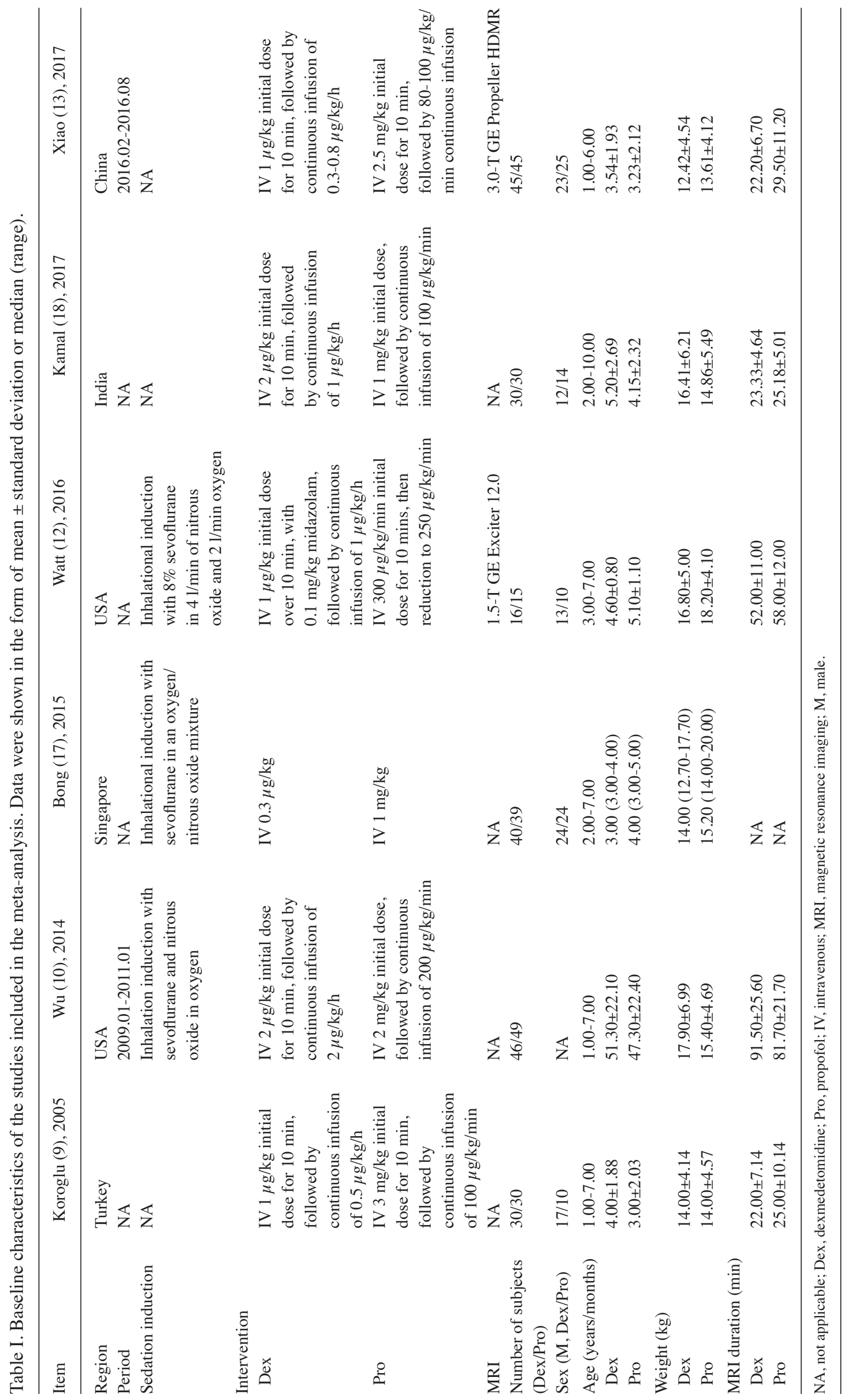


Table II. Details on sedative effects and adverse events in the studies included in the meta-analysis. Data were shown in the form of mean \pm standard deviation.

\begin{tabular}{|c|c|c|c|c|c|c|}
\hline Item & Koroglu (9), 2005 & Wu (10), 2014 & Bong (17), 2015 & Watt (12), 2016 & Kamal (18), 2017 & Xiao (13), 2017 \\
\hline \multicolumn{7}{|c|}{ Recovery time (min) } \\
\hline Dex & $27.00 \pm 19.05$ & $62.50 \pm 30.0$ & $26.00 \pm 18.00$ & $39.00 \pm 17.00$ & $9.02 \pm 2.99$ & $15.34 \pm 5.26$ \\
\hline Pro & $18.00 \pm 4.72$ & $35.70 \pm 10.8$ & $22.00 \pm 14.00$ & $27.00 \pm 9.00$ & $3.52 \pm 1.07$ & $8.43 \pm 4.51$ \\
\hline \multicolumn{7}{|c|}{ Onset of sedation time (min) } \\
\hline Dex & $11.00 \pm 4.00$ & $24.20 \pm 4.84$ & NA & $8.00 \pm 1.00$ & $7.00 \pm 1.74$ & $16.87 \pm 4.72$ \\
\hline Pro & $4.00 \pm 1.94$ & $16.30 \pm 5.54$ & NA & $8.00 \pm 2.00$ & $3.43 \pm 1.34$ & $11.51 \pm 3.92$ \\
\hline \multicolumn{7}{|c|}{ Duration of sedation (min) } \\
\hline Dex & $47.00 \pm 14.93$ & NA & $71.00 \pm 25.00$ & NA & $30.20 \pm 5.26$ & NA \\
\hline Pro & $46.00 \pm 17.59$ & NA & $70.00 \pm 28.00$ & NA & $28.60 \pm 4.61$ & NA \\
\hline \multicolumn{7}{|c|}{ Discharge time (min) } \\
\hline Dex & $39.00 \pm 24.35$ & NA & NA & $97.00 \pm 36.00$ & NA & NA \\
\hline Pro & $27.00 \pm 6.50$ & NA & NA & $91.00 \pm 27.00$ & NA & NA \\
\hline \multicolumn{7}{|c|}{ Failed sedation } \\
\hline Dex & NA & 15 & NA & NA & 9 & NA \\
\hline Pro & NA & 1 & NA & NA & 5 & NA \\
\hline \multicolumn{7}{|c|}{ Desaturation } \\
\hline Dex & 0 & 0 & NA & NA & 0 & 0 \\
\hline Pro & 4 & 2 & NA & NA & 0 & 7 \\
\hline \multicolumn{7}{|c|}{ 5-min PAED } \\
\hline Dex & NA & 18 & 9 & NA & NA & NA \\
\hline Pro & NA & 9 & 6 & NA & NA & NA \\
\hline \multicolumn{7}{|c|}{ 10-min PAED } \\
\hline Dex & NA & 16 & 5 & NA & NA & NA \\
\hline Pro & NA & 4 & 1 & NA & NA & NA \\
\hline
\end{tabular}

NA, not applicable; Dex, dexmedetomidine; Pro, propofol; min, minute; PAED, Pediatric Anesthesia Emergence Delirium.

Table III. Risk of bias assessment for all of the studies included.

\begin{tabular}{|c|c|c|c|c|c|c|c|c|}
\hline First author (year) & $\begin{array}{l}\text { Random } \\
\text { sequence } \\
\text { generation }\end{array}$ & $\begin{array}{c}\text { Allocation } \\
\text { concealment }\end{array}$ & $\begin{array}{c}\text { Blinding } \\
\text { (participants } \\
\text { and personnel) }\end{array}$ & $\begin{array}{c}\text { Blinding } \\
\text { (outcome } \\
\text { assessment) }\end{array}$ & $\begin{array}{l}\text { Incomplete } \\
\text { outcome data }\end{array}$ & $\begin{array}{l}\text { Selective } \\
\text { reporting }\end{array}$ & $\begin{array}{c}\text { Other sources } \\
\text { of bias }\end{array}$ & (Refs.) \\
\hline Koroglu (2005) & Low & Low & Low & Low & Low & Low & Low & (9) \\
\hline Wu (2014) & Unclear & Low & Low & Low & Low & Low & Low & (10) \\
\hline Bong (2015) & Low & Low & Low & Low & Low & Low & Low & (17) \\
\hline Watt (2016) & Unclear & Low & Low & Low & Low & Low & Low & (12) \\
\hline Kamal (2017) & Low & Low & Low & Low & Low & Low & Low & (18) \\
\hline Xiao (2017) & Unclear & Unclear & Low & Low & Low & Low & Low & (13) \\
\hline
\end{tabular}

Table IV. Meta-analysis results of sedative effects among the studies (Dex vs. Pro).

\begin{tabular}{|c|c|c|c|c|c|c|}
\hline \multirow[b]{2}{*}{ Outcome } & \multirow[b]{2}{*}{$\mathrm{df}$} & \multirow[b]{2}{*}{ P-value } & \multirow[b]{2}{*}{$\mathrm{RR} / \mathrm{SMD}[95 \% \mathrm{CI}]$} & \multicolumn{3}{|c|}{ Heterogeneity } \\
\hline & & & & $\mathrm{Chi}^{2}$ & $\mathrm{I}^{2}(\%)$ & P-Q test \\
\hline Recovery time & 5 & $<0.01$ & $1.11[0.55,1.66]$ & 33.62 & 85 & $<0.01$ \\
\hline Onset of sedation time & 4 & $<0.01$ & $1.44[0.77,2.12]$ & 28.33 & 86 & $<0.01$ \\
\hline Duration of sedation & 2 & 0.37 & $0.13[-0.15,0.41]$ & 0.77 & 0 & 0.68 \\
\hline Discharge time & 1 & 0.02 & $0.49[0.08,0.91]$ & 1.16 & 14 & 0.28 \\
\hline Failed sedation & 1 & 0.78 & $0.74[0.09,6.38]$ & 3.96 & 75 & 0.05 \\
\hline
\end{tabular}

Dex, dexmedetomidine; Pro, propofol; RR, risk ratio; SMD, standard mean difference; CI, confidence interval; df, degrees of freedom. 


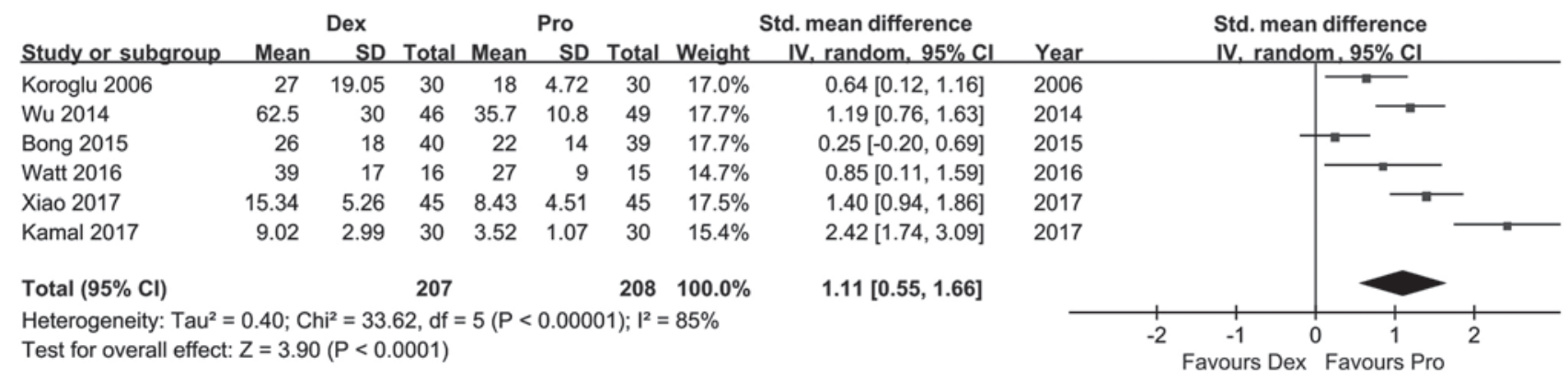

Figure 3. Forest plot of recovery time compared between Dex and Pro. Dex, dexmedetomidine; Pro, propofol; IV, inverse variance; Std., standard; SD, std. deviation; df, degrees of freedom.

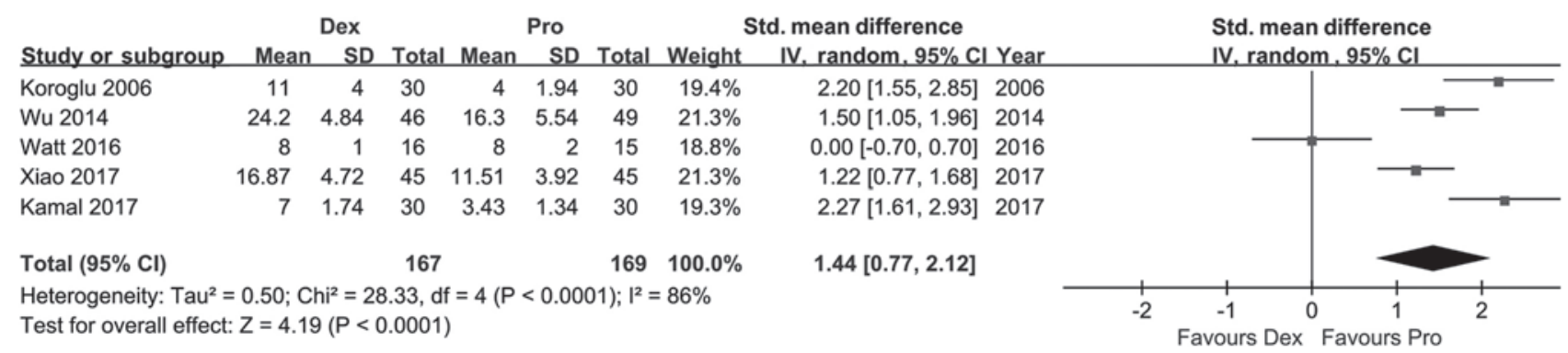

Figure 4. Forest plot of onset of sedation time compared between Dex and Pro. Dex, dexmedetomidine; Pro, propofol; IV, inverse variance; Std., standard; SD, std. deviation; df, degrees of freedom.

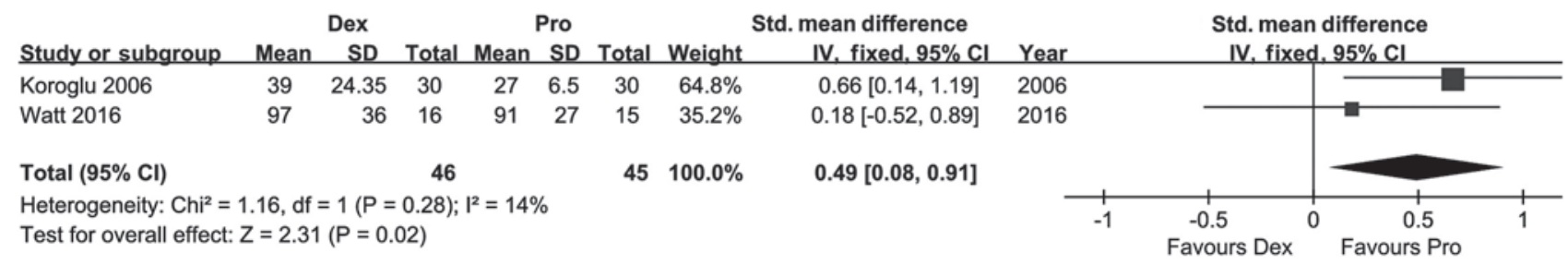

Figure 5. Forest plot of discharge time compared between Dex and Pro. Dex, dexmedetomidine; Pro, propofol; IV, inverse variance; Std., standard; SD, std. deviation; df, degrees of freedom.

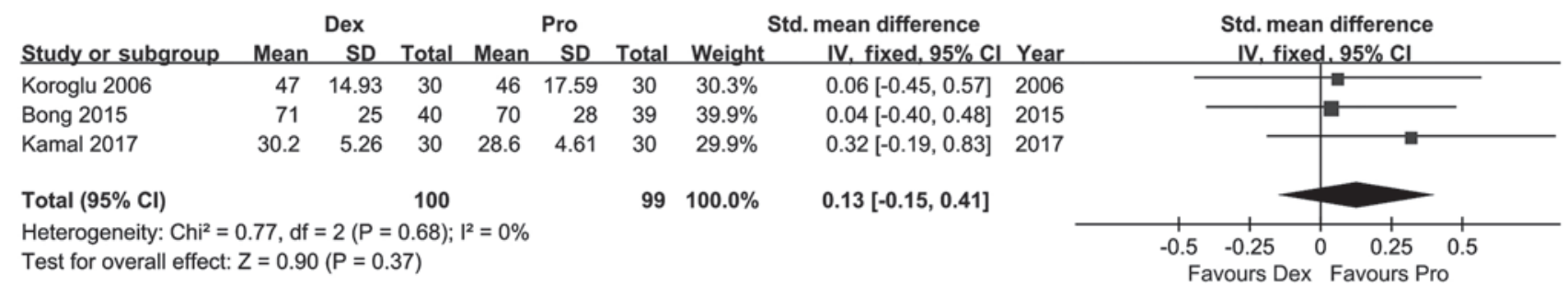

Figure 6. Forest plot of the duration of sedation compared between Dex and Pro. Dex, dexmedetomidine; Pro, propofol; IV, inverse variance; Std., standard; $\mathrm{SD}$, std. deviation; df, degrees of freedom.

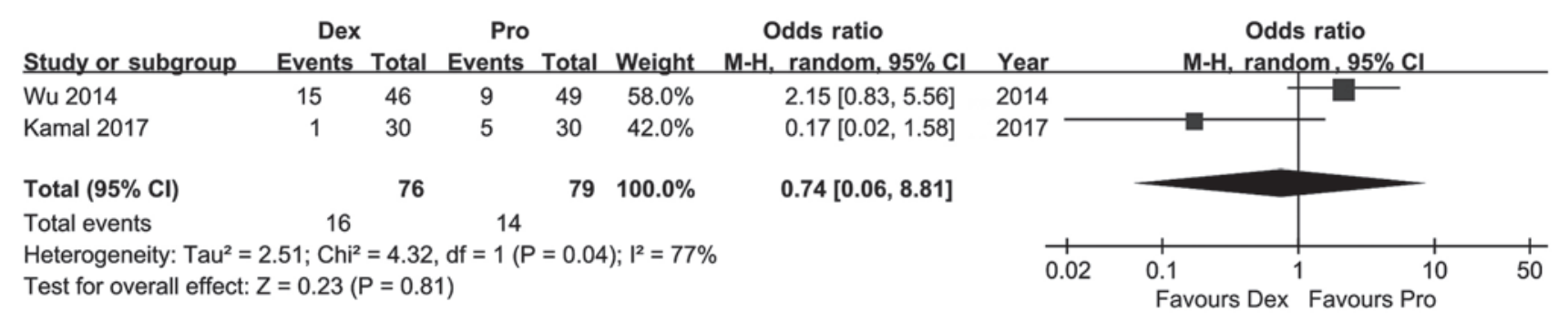

Figure 7. Forest plot of the incidence of failed sedation compared between Dex and Pro. Dex, dexmedetomidine; Pro, propofol; M-H, Mantel-Haentzel; df, degrees of freedom. 
Table V. Meta-analysis results of adverse events and MRI parameters among the studies (Dex vs. Pro).

\begin{tabular}{|c|c|c|c|c|c|c|}
\hline \multirow[b]{2}{*}{ Outcome } & \multirow[b]{2}{*}{ df } & \multirow[b]{2}{*}{ P-value } & \multirow[b]{2}{*}{ RR/SMD [95\% CI] } & \multicolumn{3}{|c|}{ Heterogeneity } \\
\hline & & & & $\mathrm{Chi}^{2}$ & $\mathrm{I}^{2}(\%)$ & P-Q test \\
\hline Desaturation & 4 & $<0.01$ & $0.11[0.02,0.55]$ & 0.31 & 0 & 0.86 \\
\hline 5-min PAED & 1 & 0.03 & $1.86[1.07,3.23]$ & 0.40 & 0 & 0.53 \\
\hline 10-min PAED & 1 & $<0.01$ & $3.49[1.50,8.16]$ & 0.01 & 0 & 0.91 \\
\hline MRI duration & 4 & 0.15 & $-0.35[-0.82,0.13]$ & 18.10 & 78 & $<0.01$ \\
\hline
\end{tabular}

Dex, dexmedetomidine; Pro, propofol; PAED, pediatric anesthesia emergence delirium; MRI, magnetic resonance imaging; min, minute; RR, risk ratio; SMD standard mean difference; CI, confidence interval; df, degrees of freedom.

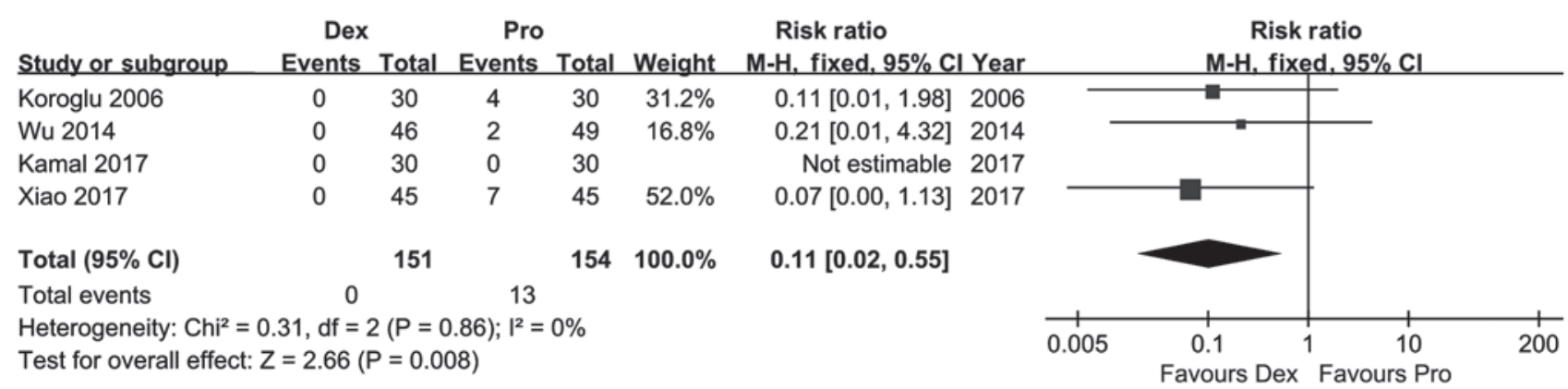

Figure 8. Forest plot of the incidence of desaturation compared between Dex and Pro. Dex, dexmedetomidine; Pro, propofol; M-H, Mantel-Haentzel; df, degrees of freedom.

\begin{tabular}{|c|c|c|c|c|c|c|c|}
\hline Study or subgroup & $\begin{array}{l}\text { Dex } \\
\text { Events }\end{array}$ & Total & $\begin{array}{r}\text { Pro } \\
\text { Events }\end{array}$ & Total & Weight & $\begin{array}{l}\text { Risk ratio } \\
\text { M-H, fixed, } 95 \% \mathrm{Cl} \text { Year }\end{array}$ & $\begin{array}{c}\text { Risk ratio } \\
\mathrm{M}-\mathrm{H} \text {, fixed, } 95 \% \mathrm{Cl}\end{array}$ \\
\hline Wu 2014 & 18 & 46 & 9 & 49 & $58.9 \%$ & $2.13[1.07,4.25] 2014$ & \\
\hline Bong 2015 & 9 & 40 & 6 & 39 & $41.1 \%$ & $1.46[0.57,3.72] 2015$ & \\
\hline Total $(95 \% \mathrm{Cl})$ & & 86 & & 88 & $100.0 \%$ & $1.86[1.07,3.23]$ & \\
\hline Total events & 27 & & 15 & & & & \\
\hline \multicolumn{7}{|c|}{$\begin{array}{l}\text { Heterogeneity: } \mathrm{Chi}^{2}=0.40, \mathrm{df}=1(P=0.53) ; I^{2}=0 \% \\
\text { Test for overall effect: } Z=2.19(P=0.03)\end{array}$} & $\begin{array}{ccc}0.7 & 1 & 1.5 \\
\text { vours Dex } & \text { Favours } \mathrm{P}\end{array}$ \\
\hline
\end{tabular}

Figure 9. Forest plot of the incidence of 5-min Pediatric Anesthesia Emergence Delirium compared between Dex and Pro. Dex, dexmedetomidine; Pro, propofol; M-H, Mantel-Haentzel; df, degrees of freedom.

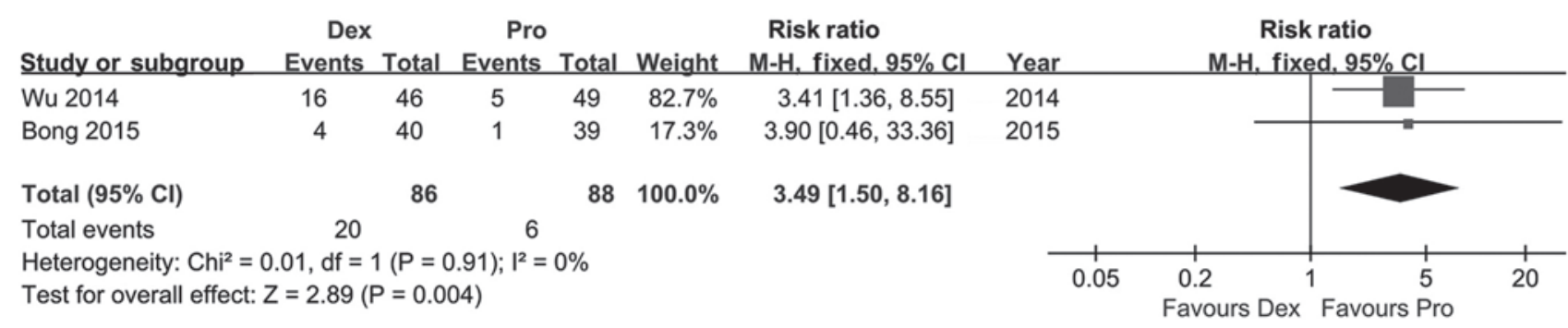

Figure 10. Forest plot of the incidence of 10-min Pediatric Anesthesia Emergence Delirium compared between Dex and Pro. Dex, dexmedetomidine; Pro, propofol; M-H, Mantel-Haentzel; df, degrees of freedom.

sooner than those with dexmedetomidine [SMD $(95 \% \mathrm{CI})$ : 0.49 (0.08, 0.91), $\mathrm{P}=0.02, \mathrm{I}^{2}=14 \%$; Fig. 5]. The pooling of data from three studies revealed no significant differences in the duration of sedation between the two interventions
[SMD (95\%CI): $0.13(-0.15,0.41), \mathrm{P}=0.37, \mathrm{I}^{2}=0 \%$; Fig. 6]. Furthermore, the incidence of failed sedation in the two groups did not differ significantly [RR $(95 \% \mathrm{CI})$ : $0.74(0.09$, 6.38), $\mathrm{P}=0.78, \mathrm{I}^{2}=75 \%$; Fig. 7]. 


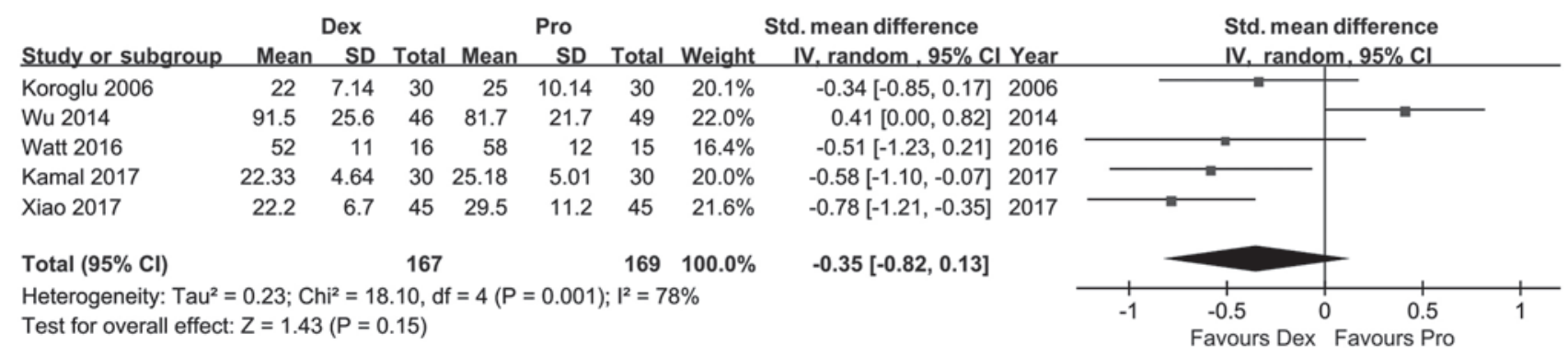

Figure 11. Forest plot of magnetic resonance imaging duration compared between Dex and Pro. Dex, dexmedetomidine; Pro, propofol; IV, inverse variance; Std., standard; SD, std, deviation; df, degrees of freedom.

Adverse events and MRI outcomes. Further outcomes, including adverse events and MRI duration, are summarized in Table V. No desaturation was reported in subjects receiving dexmedetomidine (RR [95\%CI]: 0.11 [0.02, 0.55], $\mathrm{P}<0.01$ vs propofol, $\mathrm{I}^{2}=0 \%$; Fig. 8), while propofol induced a lower incidence of 5-min (RR [95\%CI]: 1.86 [1.07, 3.23], $\mathrm{P}=0.03, \mathrm{I}^{2}=0 \%$; Fig. 9) and 10-min (RR [95\%CI]: 3.49 [1.50, 8.16], $\mathrm{P}<0.01$, $\mathrm{I}^{2}=0 \%$; Fig. 10) PAED after sedation was discontinued. The duration of scanning in the two groups was relatively similar [SMD (95\%CI): -0.35 (-0.82, 0.13), $\mathrm{P}=0.15, \mathrm{I}^{2}=78 \%$; Fig. 11], and further information is provided below in regards to sensitivity analysis. As there were $<10$ studies in total, no funnel plot analysis was performed for the detection of publication bias.

Heterogeneity and sensitivity analysis. High heterogeneity in recovery time $\left(\mathrm{I}^{2}=85 \%, \mathrm{df}=5\right)$, onset of sedation time $\left(\mathrm{I}^{2}=86 \%, \mathrm{df}=4\right)$, failed sedation $\left(\mathrm{I}^{2}=77 \%, \mathrm{df}=1\right)$ and MRI duration $\left(\mathrm{I}^{2}=78 \%, \mathrm{df}=4\right)$ was identified among the studies. Sensitivity analysis for each comparison revealed no robust changes in significance, and heterogeneity was induced when a single study was removed in the analysis of recovery time (Table VI) and onset of sedation time (Table VII). For MRI duration, exclusion of any of the trials did not change the results and heterogeneity, except for the study by Wu et al (10) $\left(\mathrm{P}<0.01, \mathrm{I}^{2}=0 \%\right.$; Table VIII). Considering the high risk of bias in the study by Xiao et al (13), all outcomes were analyzed with the exclusion of this study, which revealed a trend in the incidence of desaturation toward significance, from 0.008 to 0.07 (Table IX). The trends in any of the other outcomes were not affected.

TSA. TSA was performed for the outcomes that included $>5$ trials. It was performed to analyze the results of recovery time (Fig. 12). The TSA monitoring boundary was crossed by the cumulative $\mathrm{z}$ curve, indicating the firm evidence provided by the current results of recovery time. No boundaries were crossed in the analysis of onset of sedation time.

\section{Discussion}

The present meta-analysis summarizes the data from current RCTs comparing dexmedetomidine and propofol in pediatric patients undergoing MRI. It was indicated that propofol was associated with a shorter recovery time and onset of sedation time than dexmedetomidine. Dexmedetomidine and propofol were comparable in terms of sedation duration, the incidence of failed sedation and MRI duration. Propofol induced a lower incidence of 5-min and 10-min PAED, as well as a higher incidence of desaturation.

To date, two previous meta-analysis have been published on this topic; however, the meta-analysis by Fang et al (19) was deemed to be not as accurate. A new meta-analysis was published by our group in 2017 (11), which revealed that propofol had a shorter onset of sedation time and recovery time than dexmedetomidine. The new meta-analysis of the present study revealed similar results for these parameters, indicating better sedative effects achieved with propofol. Compared with the previous meta-analysis, the present study includes a recently published RCT (13). Furthermore, the study by Watt et al (12) was missed in the previous analysis, thus encouraging more careful attention in the selection process.

More variables were analyzed in the present analysis compared with previous meta-analysis (11). This result may be attributed to the quicker recovery from propofol than dexmedetomidine. Safety is as important as the efficacy of the interventions in clinical trials; consequently, the occurrence of desaturation, a severe condition requiring immediate treatment, was analyzed. It is thought that dexmedetomidine causes less airway collapse than propofol (12). This may explain why a higher incidence of desaturation was observed in pediatric patients who received propofol. For pediatric patients with respiratory disorders, caution should be exercised when considering the use of dexmedetomidine. To assess emergence delirium, a PAED scale was used to evaluate patients in terms of restlessness, eye contact, inconsolability, purposeful actions and consciousness of the environment $(20,21)$. Scores of $>10$ points indicated a high risk for the occurrence of emergence delirium (10). Two studies indicated a lower incidence of a PAED score of $>10$ points for propofol at 5 and 10 min after sedation, compared with dexmedetomidine. TSA is increasingly used to detect the risk for type I errors and to test the level of evidence of a meta-analysis (22). In the present study, the $\mathrm{Z}$ curve of recovery time crossed the monitoring boundary, indicating firm evidence for propofol as a preference over dexmedetomidine in terms of sedative effects.

In addition, a quality assessment of the studies included in the present meta-analysis was performed. Most of the trials were of high quality, indicating a reliable evidence 
Table VI. Sensitivity analysis of recovery time (Dex vs. Pro).

\begin{tabular}{|c|c|c|c|c|c|c|}
\hline \multirow[b]{2}{*}{ Study excluded, first author (year) } & \multirow[b]{2}{*}{ P-value } & \multirow[b]{2}{*}{ SMD $[95 \% \mathrm{CI}]$} & \multicolumn{3}{|c|}{ Heterogeneity } & \multirow[b]{2}{*}{ (Refs.) } \\
\hline & & & $\mathrm{Chi}^{2}$ & $\mathrm{I}^{2}(\%)$ & P-Q test & \\
\hline Koroglu (2005) & $<0.01$ & $1.20[0.55,1.86]$ & 31.13 & 87 & $<0.01$ & (9) \\
\hline Wu (2014) & $<0.01$ & $1.09[0.39,1.79]$ & 32.86 & 88 & $<0.01$ & $(10)$ \\
\hline Bong (2015) & $<0.01$ & $1.29[0.76,1.81]$ & 18.34 & 78 & $<0.01$ & $(17)$ \\
\hline Watt (2016) & $<0.01$ & $1.15[0.51,1.79]$ & 33.40 & 88 & $<0.01$ & (12) \\
\hline Kamal (2017) & $<0.01$ & $0.87[0.42,1.31]$ & 15.50 & 74 & $<0.01$ & (18) \\
\hline Xiao (2017) & $<0.01$ & $1.05[0.38,1.72]$ & 30.44 & 87 & $<0.01$ & (13) \\
\hline
\end{tabular}

Dex, dexmedetomidine; Pro, propofol; SMD, standard mean difference; CI, confidence interval.

Table VII. Sensitivity analysis of onset of sedation time (Dex vs. Pro).

\begin{tabular}{|c|c|c|c|c|c|c|}
\hline \multirow[b]{2}{*}{ Study excluded, first author (year) } & \multirow[b]{2}{*}{ P-value } & \multirow[b]{2}{*}{ SMD $[95 \% \mathrm{CI}]$} & \multicolumn{3}{|c|}{ Heterogeneity } & \multirow[b]{2}{*}{ (Refs.) } \\
\hline & & & $\mathrm{Chi}^{2}$ & $\mathrm{I}^{2}(\%)$ & P-Q test & \\
\hline Koroglu (2005) & $<0.01$ & $1.26[0.51,2.01]$ & 22.27 & 87 & $<0.01$ & (9) \\
\hline Wu (2014) & $<0.01$ & $1.43[0.50,2.36]$ & 28.24 & 89 & $<0.01$ & (10) \\
\hline Watt (2016) & $<0.01$ & $1.75[1.26,2.24]$ & 9.93 & 70 & 0.02 & (12) \\
\hline Kamal (2017) & $<0.01$ & $1.25[0.51,1.98]$ & 21.29 & 86 & $<0.01$ & (18) \\
\hline Xiao (2017) & $<0.01$ & $1.50[0.59,2.41]$ & 27.05 & 89 & $<0.01$ & (13) \\
\hline
\end{tabular}

Dex, dexmedetomidine; Pro, propofol; SMD, standard mean difference; CI, confidence interval.

Table VIII. Sensitivity analysis of magnetic resonance imaging time (Dex vs. Pro).

\begin{tabular}{lrcrrrr}
\hline & & & \multicolumn{3}{c}{ Heterogeneity } \\
\cline { 3 - 6 } Study excluded, first author (year) & P-value & SMD [95\% CI] & Chi $^{2}$ & $\mathrm{I}^{2}(\%)$ & P-Q test & $($ Refs.) \\
\hline Koroglu (2005) & 0.26 & $-0.35[0.97,0.26]$ & 18.07 & 83 & $<0.01$ & $(9)$ \\
Wu (2014) & $<0.01$ & $-0.58[-0.84,-0.32]$ & 1.77 & 0 & 0.62 & $(10)$ \\
Watt (2016) & 0.27 & $-0.32[-0.88,0.25]$ & 17.73 & 83 & $<0.01$ & $(12)$ \\
Kamal (2017) & 0.33 & $-0.29[-0.88,0.30]$ & 16.68 & 82 & $<0.01$ & $(18)$ \\
Xiao (2017) & 0.39 & $-0.22[-0.73,0.29]$ & 11.43 & 74 & 0.01 & $(13)$ \\
\hline
\end{tabular}

Dex, dexmedetomidine; Pro, propofol; SMD, standard mean differences; CI, confidence interval.

level of the results. Heterogeneity was identified in the outcomes of recovery time $\left(\mathrm{I}^{2}=85 \%\right)$, onset time of sedation $\left(\mathrm{I}^{2}=86 \%\right)$, failed sedation $\left(\mathrm{I}^{2}=77 \%\right)$ and MRI duration $\left(\mathrm{I}^{2}=78 \%\right)$. Only the recovery time, onset time of sedation and MRI duration were deduced from $>2$ trials. No significant change of heterogeneity emerged when sensitivity analysis was performed on recovery time and onset time of sedation. It was assumed that the high heterogeneity originated from the inconsistency in sedation details and different sample sources. Removing the study by Wu et al (10) changed the results and heterogeneity of MRI duration $(\mathrm{P}<0.00001$, $\left.\mathrm{I}^{2}=0 \%\right)$. MRI duration varied depending on body parts and sequences for scanning; however, no details of these indexes were available. It may be speculated that the high heterogeneity was caused by the diversity of body parts and scanning sequences.

The present meta-analysis had several limitations, the first of which was the relatively small number of studies for comparison, although two new studies were included compared with the previous meta-analysis by our group. Data regarding certain items in the trials were not available to us, despite numerous efforts in contacting the authors of those trials. Consequently, a thorough analysis of each variable was not possible. Furthermore, the sample size in each of the studies was relatively small. In addition, the high heterogeneity among the studies limited the credibility of the study. 
Table IX. Sensitivity analysis of incidence of desaturation (Dex vs. Pro).

\begin{tabular}{|c|c|c|c|c|c|c|}
\hline \multirow[b]{2}{*}{ Study excluded, first author (year) } & \multirow[b]{2}{*}{ P-value } & \multirow[b]{2}{*}{$\mathrm{RR}[95 \% \mathrm{CI}]$} & \multicolumn{3}{|c|}{ Heterogeneity } & \multirow[b]{2}{*}{ (Refs.) } \\
\hline & & & $\mathrm{Chi}^{2}$ & $\mathrm{I}^{2}(\%)$ & P-Q test & \\
\hline Koroglu (2006) & 0.03 & $0.10[0.01,0.78]$ & 0.32 & 0 & 0.57 & (9) \\
\hline $\mathrm{Wu}(2014)$ & 0.02 & $0.08[0.01,0.63]$ & 0.06 & 0 & 0.08 & $(10)$ \\
\hline Kamal (2017) & $<0.01$ & $0.11[0.02,0.55]$ & 0.31 & 0 & 0.86 & (18) \\
\hline Xiao (2017) & 0.07 & $0.15[0.02,1.16]$ & 0.09 & 0 & 0.76 & (13) \\
\hline
\end{tabular}

Dex, dexmedetomidine; Pro, propofol; RR, risk ratio; CI, confidence interval.

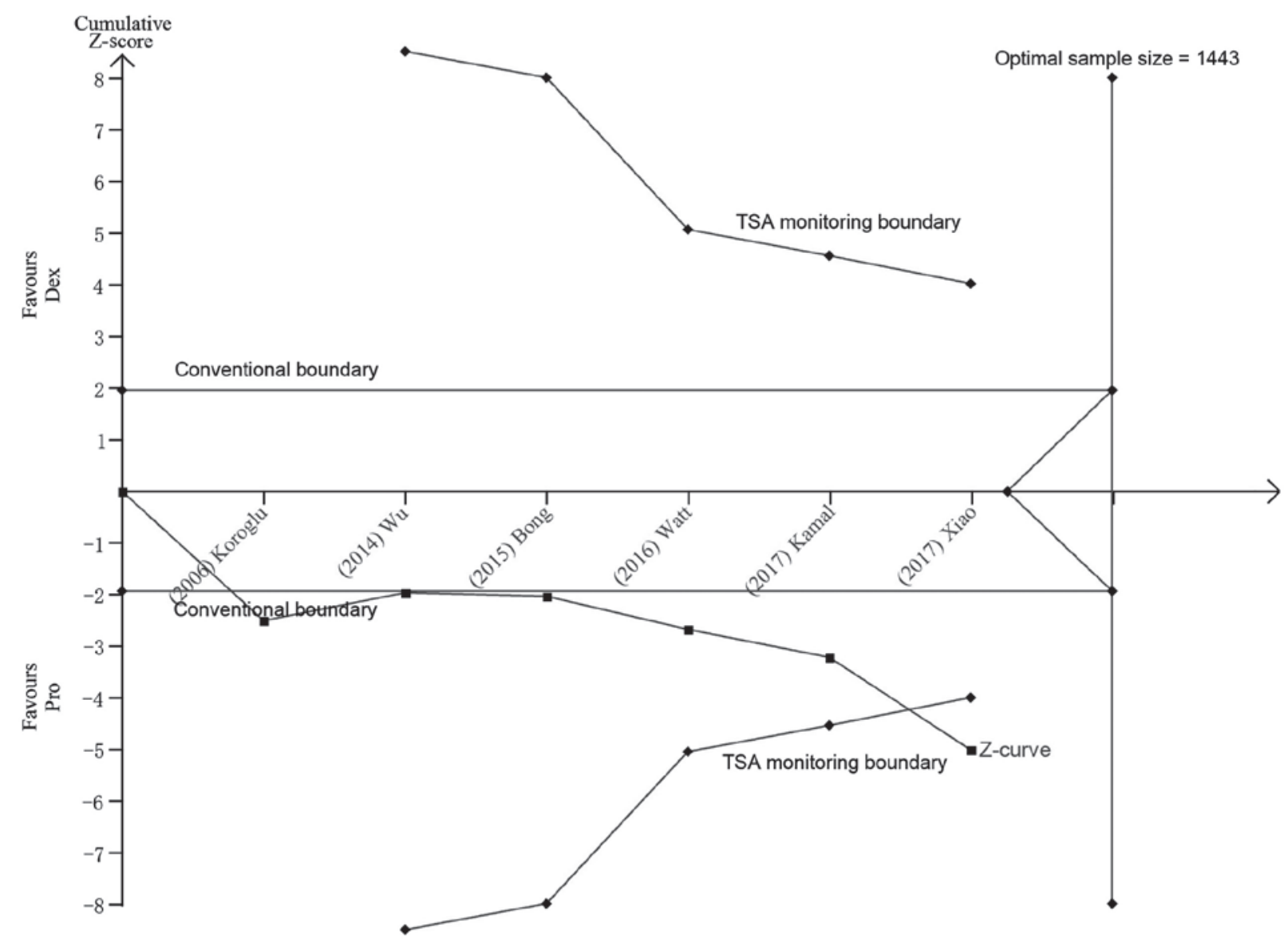

Figure 12. TSA analysis of recovery time. TSA, trial sequential analysis; Dex, dexmedetomidine; Pro, propofol.

In conclusion, the present meta-analysis indicated that dexmedetomidine and propofol had comparable sedation effects. TSA provided supportive evidence favoring propofol over dexmedetomidine in terms of shorter recovery time and onset of sedation time, as well as a faster discharge from hospital, and a lower incidence of PAED score $>10$. Propofol is recommended for sedation of pediatric patients during MRI; however, considering the possibility of desaturation, it should be used with caution.

\section{Acknowledgements}

Not applicable.

\section{Funding}

No funding was received.

\section{Availability of data and materials}

The datasets used in the meta-analysis are available from the corresponding author on reasonable request.

\section{Authors' contributions}

YT and XZ designed the study. JM and QZ performed the literature search and data extraction. JL and QZ analyzed 
the data. YT and JM drafted the manuscript. XZ revised the draft.

\section{Ethical approval and consent to participate}

Not applicable.

\section{Patient consent for publication}

Not applicable.

\section{Competing interests}

The authors declare that they have no competing interests.

\section{References}

1. Callen DJ, Shroff MM, Branson HM, Lotze T, Li DK, Stephens D and Banwell BL: MRI in the diagnosis of pediatric multiple sclerosis. Neurology 72: 961-967, 2009.

2. Moore MM, Gustas CN, Choudhary AK, Methratta ST, Hulse MA, Geeting G, Eggli KD and Boal DK: MRI for clinically suspected pediatric appendicitis: An implemented program. Pediatr Radiol 42: 1056-1063, 2012.

3. Hartman JH, Bena J, McIntyre S and Albert NM: Does a photo diarydecrease stress and anxiety in children undergoing magnetic resonance imaging? A randomized, controlled study. J Radiol Nurs 28: 122-128, 2009.

4. McJury M and Shellock FG: Auditory Noise associated with MR procedures: A review. J Magn Reson Imaging 12: 37-45, 2000.

5. Li BL, Ni J, Huang JX, Zhang N, Song XR and Yuen VM: Intranasal dexmedetomidine for sedation in children undergoing transthoracic echocardiography study-a prospective observational study. Paediatr Anaesth 25: 891-896, 2015

6. Yuen VM, Li BL, Cheuk DK, Leung MKM, Hui TWC, Wong IC, Lam WW, Choi SW and Irwin MG: A randomised controlled trial of oral chloral hydrate vs. intranasal dexmedetomidine before computerised tomography in children. Anaesthesia 72: 1191-1195, 2017.

7. Millar K, Bowman AW, Burns D, McLaughlin P, Moores T, Morton NS, Musiello T, Wallace E, Wray A and Welbury RR: Children's cognitive recovery after day-case general anesthesia: A randomized trial of propofol or isoflurane for dental procedures. Paediatr Anaesth 24: 201-207, 2014.

8. Chiaretti A, Benini F, Pierri F, Vecchiato K, Ronfani L, Agosto C, Ventura A, Genovese O and Barbi E: Safety and efficacy of propofol administered by paediatricians during procedural sedation in children. Acta Paediatr 103: 182-187, 2014.

9. Koroglu A, Demirbilek S, Teksan H, Sagir O, But AK and Ersoy MO: Sedative, haemodynamic and respiratory effects of dexmedetomidine in children undergoing magnetic resonance imaging examination: Preliminary results. Br J Anaesth 94: 821-824, 2005.
10. Wu J, Mahmoud M, Schmitt M, Hossain M and Kurth D: Comparison of propofol and dexmedetomedine techniques in children undergoing magnetic resonance imaging. Paediatr Anaesth 24: 813-818, 2014.

11. Zhou Q, Shen L, Zhang X, Li J and Tang Y: Dexmedetomidine versus propofol on the sedation of pediatric patients during magnetic resonance imaging (MRI) scanning: A meta-analysis of current studies. Oncotarget 8: 102468-102473, 2017.

12. Watt S, Sabouri S, Hegazy R, Gupta P and Heard C: Does dexmedetomidine cause less airway collapse than propofol when used for deep sedation? J Clin Anesth 35: 259-267, 2016.

13. Xiao Y, He P, Jing G, Wang Q and Wen J: Comparison of sedative effect of dexmedetomide injection and propofol injection in pediatric patients undergoing magnetic resonance imaging. Zhongguo Lin Chuang Yao Li Xue Za Zhi 33: 1764-1767, 2017 (In Chinese).

14. Knobloch K, Yoon U and Vogt PM: Preferred reporting items for systematic reviews and meta-analyses (PRISMA) statement and publication bias. J Craniomaxillofac Surg 39: 91-92, 2011.

15. Ringblom J, Wahlin I and Proczkowska M: A psychometric evaluation of the Pediatric Anesthesia Emergence Delirium scale. Paediatr Anaesth 28: 332-337, 2018.

16. Higgins JP, Altman DG, Gøtzsche PC, Jüni P, Moher D, Oxman AD, Savovic J, Schulz KF, Weeks L, Sterne JA, et al: The Cochrane Collaboration's tool for assessing risk of bias in randomised trials. BMJ 343: d5928, 2011.

17. Bong CL, Lim E, Allen JC, Choo WL, Siow YN, Teo PB and Tan JS: A comparison of single-dose dexmedetomidine or propofol on the incidence of emergence delirium in children undergoing general anaesthesia for magnetic resonance imaging. Anaesthesia 70: 393-399, 2015.

18. Kamal K, Asthana U, Bansal T, Dureja J, Ahlawat G and Kapoor S: Evaluation of efficacy of dexmedetomidine versus propofol for sedation in children undergoing magnetic resonance imaging. Saudi J Anaesth 11: 163-168, 2017.

19. Fang H, Yang L, Wang $X$ and Zhu H: Clinical efficacy of dexmedetomidine versus propofol in children undergoing magnetic resonance imaging: A meta-analysis. Int J Clin Exp Med 8: 11881-11889, 2015

20. Chandler JR, Myers D, Mehta D, Whyte E, Groberman MK, Montgomery CJ and Ansermino JM: Emergence delirium in children: A randomized trial to compare total intravenous anesthesia with propofol and remifentanil to inhalational sevoflurane anesthesia. Paediatr Anaesth 23: 309-315, 2013

21. Isik B, Arslan M, Tunga AD and Kurtipek O: Dexmedetomidine decreases emergence agitation in pediatric patients after sevoflurane anesthesia without surgery. Paediatr Anaesth 16: 748-753, 2006.

22. Wetterslev J, Jakobsen JC and Gluud C: Trial sequential analysis in systematic reviews with meta-analysis. BMC Med Res Methodol 17: 39, 2017.

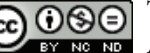

This work is licensed under a Creative Commons Attribution-NonCommercial-NoDerivatives 4.0 International (CC BY-NC-ND 4.0) License. 